\title{
Charged particle activation facility in NPI CAS and in future GANIL/SPIRAL2- NFS
}

\author{
Jaromir Mrazek ${ }^{1, *}$, Eva Simeckova ${ }^{1}$, , Radomir Behal ${ }^{1}$, Vadim Glagolev ${ }^{1}$, František Vesely ${ }^{1}$, Milan Stefanik ${ }^{1,}$, Mitja \\ Majerle $^{1,}$, Jan Novak ${ }^{1,}$, Martin Ansorge ${ }^{1,}$, Ivan Sivacek ${ }^{1}$, Jitka Vrzalova ${ }^{1}$, Xavier Ledoux ${ }^{2}$, Francois de Oliveira \\ Santos $^{2}$, Ulrich Fischer ${ }^{3}$, Axel Klix ${ }^{3}$, Marilena Avrigeanu ${ }^{4}$, and Vlad Avrigeanu ${ }^{4}$ \\ ${ }^{1}$ Nuclear Physics Institute of the Czech Academy of Sciences, 250 68, Řež, Czech Republic \\ ${ }^{2}$ GANIL CEA/DSM-CNRS/IN2P3, BP 55027, F-14076 Caen Cedex 5, France \\ ${ }^{3}$ Karlsruhe Institute of Technology, 76344 Eggenstein-Leopoldshafen, Germany \\ ${ }^{4}$ Horia Hulubei National Institute for Physics and Nuclear Engineering, P.O. Box MG-6, 077125 Bucharest-Magurele, Romania
}

\begin{abstract}
The proton, deuteron and alpha induced reactions are of a great interest for the assessment of induced radioactivity of accelerator components, targets and beam stoppers as well as isotope production for medicine and also to nuclear astrophysics. We present a new irradiation chamber for activation measurements, that forms a prolongation of long-term experimental activities using stacked-foil activation technique in NPI CAS, Řež. The chamber is based on an airlock system and is coupled to a pneumatic transfer system delivered by KIT Karlsruhe. This system is installed in GANIL/SPIRAL2-NFS and will be used for proton, deuteron and alpha particle activation measurements with long- and short-lived isotopes.
\end{abstract}

\section{Introduction}

A good knowledge of charged particle reaction cross sections is important in number of cases, we name two, here:

(a) construction, operation and decommissioning devices like ITER/DONES for IFMIF/DEMO - activation of construction materials, activation of accelerator components (targets, accelerator cavities, beamstops, etc.).

(b) improvements of the reaction models and understanding the underlying physics, that helps to make predictions in combinations of projectiles, targets and energies, where the data are missing or that are not experimentally accessible.

The experimental data are organized into large open access databases (e.g. EXFOR [1]) and are used - together with complex calculations based on theoretical models - to build open access libraries (e.g. TENDL [2]). An important part of the efforts, the underlying model parameters, obtained from interpretations are organized and kept in libraries for building databases and other use (e.g. RIPL [3]). These efforts offer a framework of systematic and consistent approach also for other investigations in nuclear reactions.

A specific case, where more experimental data are needed and a theoretical interpretation is necessary to shed light on the reaction mechanism are reactions with a deuteron projectile. Where a direct processes compete with compound ones and where a breakup contributions play an important role. Number of works were already done to coherently describe the complex activation data in

*e-mail: mrazek@ujf.cas.cz the past (e.g. [4-8]). Another theoretical challenge is a prediction of population of isomeric states. Their importance is emphasized in cases, where they decay to a different daughter nucleus than the ground state. Such experimental measurements by activation can become difficult, when the isomer half-life is shorter or comparable to the time necessary for manipulation with the activated sample.

While the number of systematic measurements and theoretical explanations were performed in the last decades, that have improved our knowledge and extended the available databases, there are still reactions, where the actual systematic measurements are difficult and thus very limited or non-existing. One of the important cases are the reactions leading to short-lived products. The aim of this paper is to introduce the complex system for measurements of both short-lived and long-lived isotopes in NFS [9] (Neutrons For Science) laboratory in GANILSPIRAL2.

\section{Activation setup in NPI CAS}

Nuclear Physics Institute of the Academy of Sciences of Czech Republic operates two cyclotrons: light particle, variable energy $\mathrm{U}-120 \mathrm{M}$ cyclotron (currents from $\mathrm{nA}$ to tens of $\mu \mathrm{A}$ ) and a new high current (up to $300 \mu \mathrm{A}$ ) proton cyclotron TR24 (see the table 1). The operation of the cyclotrons is supported by the project CANAM (Center of Accelerators and Nuclear Analytical Methods) [10] of MEYS and RDI OP of EU.

The traditional charged particle activation setup used in NPI CAS consists of a stack of foils to be measured that are interleaved by monitoring foils (e.g. aluminium 

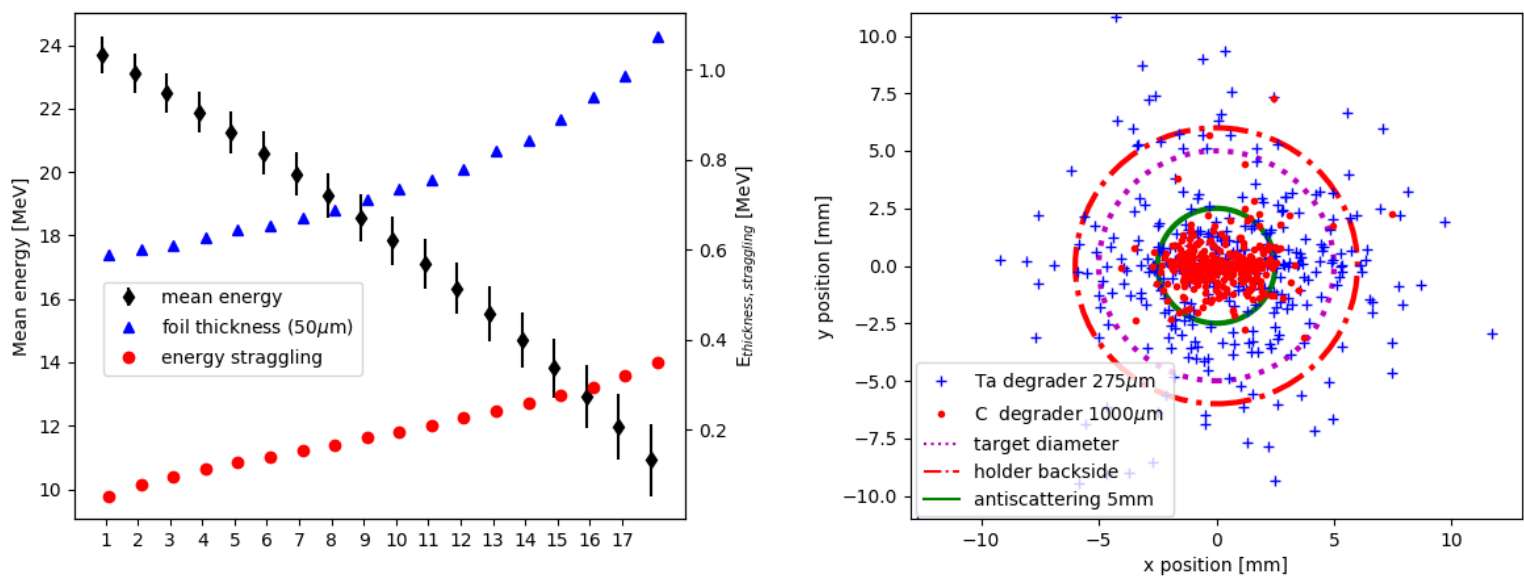

Figure 1. Left panel: a stacked foil simulation - an illustrative case of a proton beam on stack of $50 \mu \mathrm{m}{ }^{n a t} \mathrm{Fe}$ foils. Foil number is on $\mathrm{x}$-axis, mean energy in the foil (black diamonds) is on the left y-axis), a foil thickness in terms of energy (blue triangles) and an energy straggling (red circles) are on the right-side y-axis. Right panel: A proton beam deposition simulation for Irradiation Chamber beamtarget setup after $1 \mathrm{~mm}$ thick carbon degrader. The deposition is drawn at the backside of the sample holder. The distance between the degrader and the activation foil was $28 \mathrm{~mm}$. The beam after the carbon degrader looses $5 \mathrm{MeV}$ and is concentrated at the center (red dots). The beam spread for tantalum degrader (blue crosses) is shown for a comparison. The anti-scattering size is only depicted (a green circle) and was not included in the simulation.

Table 1. Beam energies in [MeV] of U-120M and TR24 cyclotrons (the second row is for TR24) in NPI CAS.

\begin{tabular}{llll}
\hline protons & deuterons & ${ }^{3} \mathrm{He}$ & ${ }^{4} \mathrm{He}$ \\
\hline $6-38$ & $11-20$ & $16-53$ & $22-40$ \\
$18-24$ & - & - & -
\end{tabular}

[4]), that (a) make sure that there is no unobserved particle exchange between neighbouring foils and that (b) the complete chain of procedures - from the beam energy and current measurement till detector efficiency calibration - is correct.

This method enables a simultaneous measurement of a number of data points. The energy losses and energy straggling have an impact on precision of (especially) low energy data points (see the left panel of figure 1). In measurements of short-lived isotopes with a stacked foils method, one needs to find a compromise between handling possibility of the accumulated activity and accessibility of shorter-lived isotopes. The stacked foils method was successfully applied in a number of experiments ([4-8]).

\section{NFS at GANIL/SPIRAL2}

A laboratory NFS (Neutrons For Science) in GANIL/SPIRAL2 was already presented elsewhere [9]. Despite its name, NFS laboratory will open new opportunities also in activation by charged particles. Initially, light ion beams ( $\mathrm{p}, \mathrm{d}, \alpha)$ are expected to be available with a maximum current $50 \mu \mathrm{A}$ (a limit allowed for NFS, the maximum reachable current is $5 \mathrm{~mA}$ ). The available energies are displayed in the table 2.

In the next section, we present the system for irradiation by charged particles for SPIRAL2/NFS, that was built
Table 2. Beam energies in $[\mathrm{MeV}]$ of LINAC accelerator of GANIL/SPIRAL2.

\begin{tabular}{llll}
\hline protons & deuterons & ${ }^{3} \mathrm{He}$ & ${ }^{4} \mathrm{He}$ \\
\hline $0.75-33$ & $1.5-40$ & $2.25-72$ & $3-80$
\end{tabular}

within a program SPIRAL2-CZ, that is a framework of a Czech participation in GANIL/SPIRAL2.

\section{Irradiation Chamber system}

The need of construction of the new system for charged particle activation stems primarily from the limitations of deuteron beams in NPI CAS (see the tables 1,2) and a simultaneous need of extension of the experimental possibilities. The demands for the construction of the system were based on long term experiences from NPI CAS and specific mode of operation in GANIL/SPIRAL2 NFS. A transport of a stack of foils between a beam in vacuum and storage was initially requested.

Further demands were defined, as a new technological solution and thus new abilities appeared:

- an irradiation of a single foil to measure short half-lives

- a sub-minute transport time from the irradiation to the detector

- a possibility of the fast change of the beam energy

- a basic information on the beam quality on-line

- a remote operation due to imposed safety restrictions

The selected solution was based on an airlock system coupled to a pneumatic transport system. The airlock system (in figure 2) is composed of two vacuum chambers: 


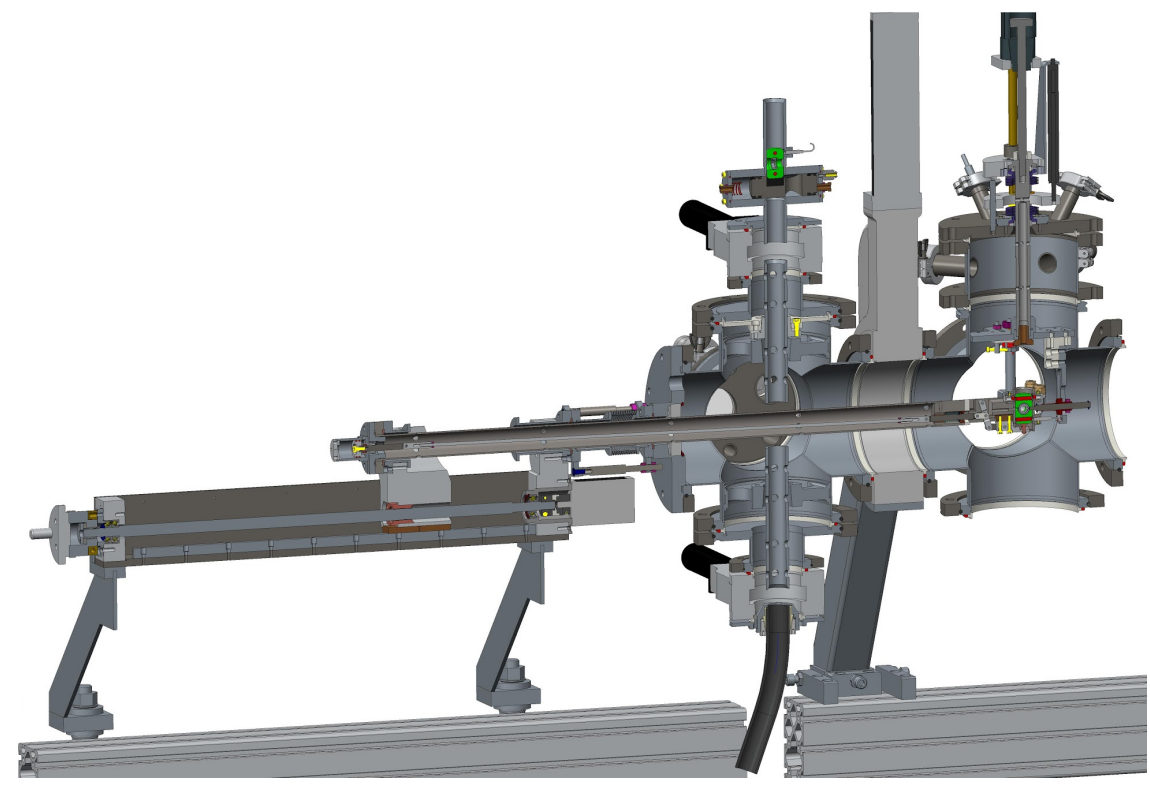

Figure 2. The Irradiation Chamber. It is composed of two vacuum chambers separated by an interlock valve (light gray). The rightmost part is permanently in the beamline and it contains a beam-target system. The left part contains a system for receiving/sending samples and is equipped with a manipulator. Two sample holders are depicted in green - one is waiting for receiving (in the middle) and the other is in the beam position (on the right).

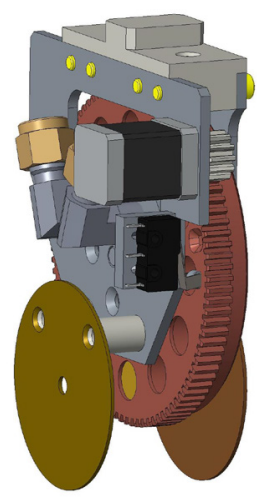

Figure 3. Beam-target system. Beam direction is from the left, collimator, twelve position degrader, anti-scattering-electrode.

one is equipped by a beam-target system and is permanently a part of the NFS beam-line. The other one is equipped by a sample receive/send system and a manipulator, that transfers a sample to/from the beam-target system of the first chamber.

\section{Beam-target system}

The beam-target system (see figure 3) consists of

- an entrance collimator

- a cooled rotating degrader with twelve selectable positions

- an anti-scattering collimator - electrode

- a sample lock system with a suspension

- a cooled Faraday cup

The beam is designed to have pencil-like characteristics with $4 \mathrm{~mm}$ diameter of the core. The entrance collimator is isolated and has a current readout, so that the information on beam position/focus change is available online.
The degrader will allow for a fast change of beam energy (as the time needed for the energy change of the accelerator and subsequent magneto-optic tuning is yet unknown ) at the expense of the energy (and angular - see right panel of figure 1) straggling. The degrader is connected to the same cooling loop as the Faraday cup and the isolation better than $500 \mathrm{M} \Omega$ is maintained. The degrader has its own electrical readout electrode. The third element downstream is an anti-scattering collimator-electrode, that has several functions: (a) to remove eventual particles scattered by collimator or degrader to large angles (angular straggling, see figure 1), (b) to pose as an electrode for the sample+Faraday cup block, to repel eventual kicked-out electrons in Faraday cup measurement, (c) keep the electrons coming from the degrader material on the degrader side or deflect them by a pair of magnets that are installed around the collimator hole.

Collimator diameters are $5 \mathrm{~mm}$ both.

Cooling system was tested successfully with alcohol, but for safety constraints it will operate with much more viscous ethylene-glycol. The expected typical power on the system is in the order of Watts, the system was tested at $40 \mathrm{~W}$.

The beam-target system is remotely retractable from the beam, so that after an irradiation, another experiment can take a place without a need of operator intervention.

\section{Manipulator system and a sample holder}

The second - send/receive - chamber is based on a 6-way cross with five valves. It is equipped by a manipulator arm, that can be positioned in the range of $0-350 \mathrm{~mm}$. Two valves operate when a sample is needed to be received and sent, other two valves serve for the venting and evacuat- 


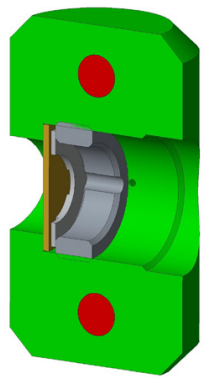

Figure 4. Sample holder - a cut. The red circles are the magnets (see the text), the foil (in brown) is fastened by a steel bolt (in gray).

ing. The interlock valve (between the chambers) can be operated, when the same pressure is on its both sides.

For practical and operational speed reasons, the samples are received from top (see the middle part of figure 2) and sent out (also exploiting the gravity) downwards. To use the stacked-foil method, the key property of a manipulator system is to align the sample holder not only to the beam axis, but also direction-wise. To ensure this, we decided for the design of the sample holder as shown in figure 4. The sample holder is from aluminium, $19 \mathrm{~mm}$ in diameter and $40 \mathrm{~mm}$ long. It is pierced through so that it can house a foil (a stack of foils) of $15 \mathrm{~mm}$ diameter. The inner diameter of the steel bolt fixing the foil(s) is $11 \mathrm{~mm}$. The correct orientation in the beam (or detector) is ensured by two (symmetrically placed) magnets fixed in the body of the sample holder. The orientation of the sample - once it reaches the manipulator (in the airlock system or at the detector) - is immediate.

\section{Further operation details}

The operation of the airlock system has a slow phase of evacuating down to $10^{-5}$ mbar and a fast pressurized $\mathrm{N}_{2}$ venting using pressure guards to avoid eventual critical conditions for the valves.

The Faraday cup is inclined by approx. $30^{\circ}$ from the beam axis towards the manipulator. When the sample is being translated to the beam position, it comes into an electrical and thermal contact with the Faraday cup and then the Faraday rotates to the beam axis. This way the cooling and charge readout of the sample holder and the Faraday cup is connected.

There were performed several tests on U-120M cyclotron of NPI CAS. These tests allowed to solve number of issues, define degrader material (see figure 1) and proved the possibility to transfer the sample from the beam position to the HPGe detector (including venting) well bellow one minute. The operational tests were performed with proton beam and ${ }^{n a t} \mathrm{Fe}$ foils and the measured data follow the previous experimental data and TENDL curves of different reactions $\left({ }^{n a t} \mathrm{Fe}(\mathrm{p}, \mathrm{x}){ }^{52} \mathrm{Mn},{ }^{n a t} \mathrm{Fe}(\mathrm{p}, \mathrm{x}){ }^{53 m+g} \mathrm{Fe}\right)$.

\section{Pneumatic Transfer System}

The Pneumatic Transport System (PTS) for neutron irradiations is based on a system of TU Dresden and it was delivered by KIT Karlsruhe. Sample holders were made of plastic (lighter than aluminium) and the activation foil was located on top of the sample holder.
The PTS system was adapted for the charge particle sample holders: it was equipped with air-brakes at every ending point to keep the deceleration of the aluminium sample holders at reasonable limits.

The control software was built in LabView, that communicates with PLC system of the PTS system, software of the PTS, controllers of the IC system and control system of the HPGe detector positioning.

\section{Summary}

The complex system of Irradiation Chamber (IC) and PTS for charged particle activation was installed in NFS laboratory of GANIL/SPIRAL2 facility, where the access is open and excellence based.

The Irradiation Chamber was built, tested and installed also with the efforts of J.Diviš, J.Záluský, J.Červeňanský, L.Király, S.Slovák, M.Gschray, J.Gabrhel, M.Götz. Authors are indebted to GANIL/SPIRAL2 technical staff, that provided help and cooperation during various phases of the project. The authors acknowledge the MEYS project SPIRAL2-CZ, CZ.02.1.01/0.0/0.0/16_013/0001679.

\section{References}

[1] N. Otuka, E. Dupont, V. Semkova, B. Pritychenko, A. Blokhin, M. Aikawa, S. Babykina, M. Bossant, G. Chen, S. Dunaeva et al., Nuclear Data Sheets 120, 272 (2014)

[2] A. Koning, D. Rochman, Nuclear Data Sheets 113, 2841 (2012), special Issue on Nuclear Reaction Data

[3] R. Capote, M. Herman, P. Obložinský, P. Young, S. Goriely, T. Belgya, A. Ignatyuk, A. Koning, S. Hilaire, V. Plujko et al., Nuclear Data Sheets 110, 3107 (2009), special Issue on Nuclear Reaction Data

[4] P. Bém, E. Šimečková, M. Honusek, U. Fischer, S.P. Simakov, R.A. Forrest, M. Avrigeanu, A.C. Obreja, F.L. Roman, V. Avrigeanu, Phys. Rev. C 79, 044610 (2009)

[5] M. Avrigeanu, V. Avrigeanu, P. Bém, U. Fischer, M. Honusek, A.J. Koning, J. Mrázek, E. Šimečková, M. Štefánik, L. Závorka, Phys. Rev. C 88, 014612 (2013)

[6] M. Avrigeanu, V. Avrigeanu, P. Bém, U. Fischer, M. Honusek, K. Katovsky, C. Mănăilescu, J. Mrázek, E. Šimečková, L. Závorka, Phys. Rev. C 89, 044613 (2014)

[7] M. Avrigeanu, E. Šimečková, U. Fischer, J. Mrázek, J. Novak, M. Štefánik, C. Costache, V. Avrigeanu, Phys. Rev. C 94, 014606 (2016)

[8] E. Šimečková, P. Bém, M. Honusek, M. Štefánik, U. Fischer, S.P. Simakov, R.A. Forrest, A.J. Koning, J.C. Sublet, M. Avrigeanu et al., Phys. Rev. C 84, 014605 (2011)

[9] X. Ledoux, M. Aïche, M. Avrigeanu, V. Avrigeanu, L. Audouin, E. Balanzat, B. Ban-détat, G. Ban, G. Barreau, E. Bauge et al., Nuclear Data Sheets 119, $353(2014)$

[10] http://canam.ujf.cas.cz 